\title{
Reactivity of Aluminum Complexes of Redox-Active Ligand toward N-Heterocyclic Carbene and Its Thione
}

\author{
Vladimir G. Sokolov, ${ }^{a}$ Tatyana S. Koptseva, ${ }^{a}$ Roman V. Rumyantcev, ${ }^{a}$ \\ Xiao-Juan Yang, ${ }^{\mathrm{b}}$ Yanxia Zhao, ${ }^{\mathrm{b}}$ Igor L. Fedushkin ${ }^{* a, b}$ \\ ${ }^{\text {a }}$ G.A. Razuvaev Institute of Organometallic Chemistry of Russian Academy of Sciences, \\ Tropinina str. 49, 603950, Nizhny Novgorod, Russian Federation \\ ${ }^{\mathrm{b}}$ College of Chemistry and Materials Science, Northwest University, Xi'an 710069, China
}

X-ray crystallographic data for compounds 3-6. The X-ray diffraction data were collected on a Bruker D8 Quest Photon (for 3 and 4) and Oxford Xcalibur Eos (5, 6) diffractometers (Mo-K $\mathrm{K}_{\alpha}$ radiation, $\omega$-scan technique, $\lambda=0.71073 \AA$ ). The intensity data were integrated by SAINT $(\mathbf{2}, \mathbf{3})^{1}$ and CrysAlisPro $(\mathbf{5}, \mathbf{6})^{2}$ programs. The structures were solved by using a dualspace algorithm (3 and 5$)^{3}$ and direct methods (4 and 6). All structures were refined on $\mathrm{F}_{\mathrm{hkl}}{ }^{2}$ using SHELXTL package. ${ }^{4}$ Samples of $\mathbf{4}$ and $\mathbf{6}$ were refined as two-component twins (HKLF4 and HKLF5 were used for final refinement of complex 4 and $\mathbf{6}$, respectively). The refined BASF parameter for the prevailing component equals 0.5438(1) (for 4) and 0.6181(1) (6). All non-hydrogen atoms were refined anisotropically. All hydrogen atoms except $\mathrm{H}(1)$ in complex 6 were placed in calculated positions and were refined in the riding model $\left(U_{\text {iso }}(H)=\right.$ $1.5 \mathrm{U}_{\text {eq }}(\mathrm{C})$ in $\mathrm{CH}_{3}$-groups and $\mathrm{U}_{\mathrm{iso}}(\mathrm{H})=1.2 \mathrm{U}_{\mathrm{eq}}(\mathrm{C})$ in other groups). The hydrogen atom $\mathrm{H}(1)$ in complex 6 was localized from the difference Fourier synthesis and refined in the isotropic approximation. SADABS $(\mathbf{3}, \mathbf{4})^{5}$ and and SCALE3 ABSPACK scaling algorithm into CrysAlisPro $(\mathbf{5}, \mathbf{6})^{2}$ were used to perform absorption corrections. The crystals of 3-6 contains solvate molecules. There are 0.5 toluene molecule per one complex molecule in $\mathbf{3}, 1.5$ toluene molecules in 4, two DME molecules in 5 and 0.5 toluene molecule in $\mathbf{6}$. The main crystallographic data and structure refinement details for 3-6 are presented in Table 1. CCDC 1949240 (3), 1949241 (4), 1949242 (5) and 1949243 (6) contain the supplementary crystallographic data. These data can be obtained free of charge from The Cambridge Crystallographic Data Centre via ccdc.cam.ac.uk/structures/.

DFT Calculations. Structure optimization for the model compounds (dpp-bian)Al-Al(dppbian) (1), (dpp-bian)AlH(THF) (2), (dpp-bian)(NHC)Al-Al(dpp-bian) (3) and (dppbian)AlH(NHC) (6) were carried out at the DFT (B3LYP) level with a 6-31G*6,7 basis set using the Gaussian 09 program. ${ }^{8}$ 
(1) SAINT. Data Reduction and Correction Program v. 8.37A, Bruker AXS, Madison, Wisconsin, USA, 2017.

(2) Rigaku Oxford Diffraction, CrysAlisPro Software system, v. 1.171.38.46, Rigaku Corporation, Oxford, UK, 2015.

(3) Sheldrick, G. SHELXT - Integrated space-group and crystal-structure determination. Acta Cryst. 2015, A 71, 3-8.

(4) Sheldrick, G. Crystal structure refinement with SHELXL. Acta Cryst. 2015, C 71, 3-8.

(5) Krause, L.; Herbst-Irmer, R.; Sheldrick, G.M.; Stalke, D. Comparison of silver and molybdenum microfocus X-ray sources for single-crystal structure determination. J. Appl. Cryst. 2015, 48, 3-10.

(6) Becke, A. D. Density-functional thermochemistry. III. The role of exact exchange. J. Chem. Phys. 1993, 98, 5648-5652.

(7) Lee, C.; Yang W.; Parr, R. G. Development of the Colle-Salvetti correlation-energy formula into a functional of the electron density. Phys. Rev. B., 1988, 37, 785-789.

(8) Gaussian 09, Revision C.1, Frisch, M. J.; Trucks, G. W.; Schlegel, H. B.; Scuseria, G. E.; Robb, M. A.; Cheeseman, J. R.; Scalmani, G.; Barone, V.; Mennucci, B.; Petersson, G. A.; Nakatsuji, H.; Caricato, M.; Li, X.; Hratchian, H. P.; Izmaylov, A. F.; Bloino, J.; Zheng, G.; Sonnenberg, J. L.; Hada, M.; Ehara, M.; Toyota, K.; Fukuda, R.; Hasegawa, J.; Ishida, M.; Nakajima, T.; Honda, Y.; Kitao, O.; Nakai, H.; Vreven, T.; Montgomery, Jr., J. A.; Peralta, J. E.; Ogliaro, F.; Bearpark, M.; Heyd, J. J.; Brothers, E.; Kudin, K. N.; Staroverov, V. N.; Kobayashi, R.; Normand, J.; Raghavachari, K.; Rendell, A.; Burant, J. C.; Iyengar, S. S.; Tomasi, J.; Cossi, M.; Rega, N.; Millam, J. M.; Klene, M.; Knox, J. E.; Cross, J. B.; Bakken, V.; Adamo, C.; Jaramillo, J.; Gomperts, R.; Stratmann, R. E.; Yazyev, O.; Austin, A. J.; Cammi, R.; Pomelli, C.; Ochterski, J. W.; Martin, R. L.; Morokuma, K.; Zakrzewski, V. G.; Voth, G. A.; Salvador, P.; Dannenberg, J. J.; Dapprich, S.; Daniels, A. D.; Farkas, Ö.; Foresman, J. B.; Ortiz, J. V.; Cioslowski, J.; Fox, D. J. Gaussian, Inc., Wallingford CT, 2009. 
Table 1. Crystal data and structure refinement details for compounds 3-6.

\begin{tabular}{|c|c|c|c|c|}
\hline & 3 & 4 & 5 & 6 \\
\hline Empirical Formula & $\mathrm{C}_{86.50} \mathrm{H}_{104} \mathrm{Al}_{2} \mathrm{~N}_{6}$ & $\mathrm{C}_{104.50} \mathrm{H}_{134} \mathrm{Al}_{2} \mathrm{~N}_{8} \mathrm{~S}_{2}$ & $\mathrm{C}_{102} \mathrm{H}_{140} \mathrm{~A}_{2} \mathrm{~N}_{8} \mathrm{O}_{4} \mathrm{~S}$ & $\mathrm{C}_{50.50} \mathrm{H}_{65} \mathrm{AlN}_{4}$ \\
\hline M & 1281.71 & 1620.27 & 1628.23 & 755.04 \\
\hline $\mathrm{T} / \mathrm{K}$ & $100(2)$ & $100(2)$ & $100(2)$ & $100(2)$ \\
\hline Crystal System & monoclinic & triclinic & Triclinic & triclinic \\
\hline Space Group & $\mathrm{P} 2{ }_{1} / \mathrm{n}$ & $\mathrm{P}-1$ & P-1 & $\mathrm{P}-1$ \\
\hline $\mathrm{a} / \AA ̊$ & $12.4899(4)$ & $13.247(2)$ & $12.73595(19)$ & $12.8299(5)$ \\
\hline $\mathrm{b} / \AA$ & $27.2515(9)$ & $16.392(4)$ & $18.5631(3)$ & $18.2822(4)$ \\
\hline $\mathrm{c} / \AA$ & $21.6359(7)$ & $23.630(4)$ & $19.6627(3)$ & $20.0455(4)$ \\
\hline$\alpha / \operatorname{deg}$ & 90 & $81.347(4)$ & $79.6121(12)$ & $93.108(2)$ \\
\hline$\beta / \operatorname{deg}$ & $92.4570(16)$ & $73.784(3)$ & $85.9195(12)$ & $96.587(2)$ \\
\hline$\gamma / \operatorname{deg}$ & 90 & $66.231(3)$ & $86.5802(12)$ & $107.085(3)$ \\
\hline $\mathrm{V} / \AA^{3}$ & $7357.4(4)$ & $4504.8(14)$ & $4555.67(12)$ & $4445.5(2)$ \\
\hline Z & 4 & 2 & 2 & 4 \\
\hline $\mathrm{d}_{\text {cald }} / \mathrm{Mg} \mathrm{m}^{-3}$ & 1.157 & 1.195 & 1.187 & 1.128 \\
\hline$\mu(\mathrm{Mo} \mathrm{K} \alpha) / \mathrm{mm}^{-1}$ & 0.089 & 0.132 & 0.111 & 0.084 \\
\hline $\mathrm{F}(000)$ & 2764 & 1750 & 1764 & 1636 \\
\hline Crystal Size/mm & $0.33 \times 0.17 \times 0.11$ & $0.36 \times 0.23 \times 0.015$ & $0.27 \times 0.20 \times 0.17$ & $0.74 \times 0.40 \times 0.21$ \\
\hline$\theta$ range/deg & $1.994-25.027$ & $2.206-24.676$ & $2.834-26.022$ & $2.824-26.029$ \\
\hline \multirow{3}{*}{$\mathrm{h}, \mathrm{k}, \mathrm{l}$} & $-14 \leq \mathrm{h} \leq 14$ & $-15 \leq h \leq 15$ & $-15 \leq h \leq 15$ & $-15 \leq \mathrm{h} \leq 15$ \\
\hline & $-32 \leq \mathrm{k} \leq 32$ & $-19 \leq \mathrm{k} \leq 19$ & $-22 \leq \mathrm{k} \leq 22$ & $-22 \leq \mathrm{k} \leq 22$ \\
\hline & $-25 \leq 1 \leq 25$ & $-27 \leq 1 \leq 27$ & $-24 \leq 1 \leq 23$ & $-24 \leq 1 \leq 24$ \\
\hline $\begin{array}{l}\text { Reflections } \\
\text { Collect. }\end{array}$ & 65052 & 35325 & 59992 & 31921 \\
\hline Indep. Reflections & 12887 & 15027 & 17717 & 31921 \\
\hline $\mathrm{R}_{\text {int }}$ & 0.0654 & 0.0940 & 0.0363 & 0.0499 \\
\hline Data/Restr./Param. & 12887 / 19 / 906 & 15027 / 43 / 1094 & 17717 / 0 / 1086 & $31921 / 104 / 1075$ \\
\hline GooF & 1.015 & 1.054 & 1.030 & 0.991 \\
\hline $\mathrm{R}_{1} / \mathrm{wR}_{2}(\mathrm{I}>2 \sigma(\mathrm{I}))$ & $0.0477 / 0.1327$ & $0.0674 / 0.1609$ & $0.0436 / 0.0939$ & $0.0503 / 0.1113$ \\
\hline $\mathrm{R}_{1} / \mathrm{wR}_{2}$ (all data) & $0.0693 / 0.1462$ & $0.0925 / 0.1745$ & $0.0633 / 0.1016$ & $0.0755 / 0.1164$ \\
\hline $\begin{array}{l}\text { Larg. Diff. Peak } \\
\text { and Hole/e } \AA^{-3}\end{array}$ & $0.332 /-0.356$ & $0.554 /-0.559$ & $0.332 /-0.267$ & $0.350 /-0.335$ \\
\hline
\end{tabular}


Table 2. Selected bond lengths $[\AA]$ and angles $\left[{ }^{\circ}\right]$ for complexes 3-6.

\begin{tabular}{|c|c|c|c|c|}
\hline Bond & 3 & 4 & 5 & 6 \\
\hline $\mathrm{Al}(1)-\mathrm{N}(1)$ & $1.8796(16)$ & $1.873(4)$ & $1.8989(14)$ & $1.8715(17)$ \\
\hline $\mathrm{Al}(1)-\mathrm{N}(2)$ & $1.8889(16)$ & $1.866(4)$ & $1.8943(15)$ & $1.8759(17)$ \\
\hline $\mathrm{Al}(2)-\mathrm{N}(3)$ & $1.8743(16)$ & $1.894(4)$ & $1.9025(15)$ & \\
\hline $\mathrm{Al}(2)-\mathrm{N}(4)$ & $1.8535(17)$ & $1.854(4)$ & $1.9014(14)$ & \\
\hline $\mathrm{N}(1)-\mathrm{C}(1)$ & $1.415(2)$ & $1.408(5)$ & $1.400(2)$ & $1.400(2)$ \\
\hline $\mathrm{N}(2)-\mathrm{C}(2)$ & $1.391(2)$ & $1.393(5)$ & $1.398(2)$ & $1.409(2)$ \\
\hline$C(1)-C(2)$ & $1.369(3)$ & $1.364(6)$ & $1.370(2)$ & $1.369(3)$ \\
\hline $\mathrm{N}(3)-\mathrm{C}(37)$ & $1.407(2)$ & $1.396(5)$ & $1.397(2)$ & \\
\hline $\mathrm{N}(4)-\mathrm{C}(38)$ & $1.386(2)$ & $1.405(5)$ & $1.414(2)$ & \\
\hline$C(37)-C(38)$ & $1.366(3)$ & $1.360(7)$ & $1.370(2)$ & \\
\hline $\mathrm{Al}-\mathrm{C}(\mathrm{carb})$ & $2.094(2)$ & $2.071(4)$ & $\begin{array}{l}2.0762(17), \\
2.0710(17)\end{array}$ & $2.057(2)$ \\
\hline $\mathrm{Al}(1)-\mathrm{S}(1)$ & & $2.2062(16)$ & $2.2760(6)$ & \\
\hline $\mathrm{Al}(2)-\mathrm{S}(1)$ & & $2.2397(16)$ & $2.2670(6)$ & \\
\hline $\mathrm{Al}(1)-\mathrm{S}(2)$ & & $2.3341(16)$ & & \\
\hline $\mathrm{Al}-\mathrm{H}(1)$ & & & & $1.524(18)$ \\
\hline \multicolumn{5}{|l|}{ Angle } \\
\hline $\mathrm{N}(1)-\mathrm{Al}(1)-\mathrm{N}(2)$ & $91.81(7)$ & $91.58(15)$ & $90.03(6)$ & $91.48(7)$ \\
\hline $\mathrm{N}(4)-\mathrm{Al}(2)-\mathrm{N}(3)$ & $91.10(7)$ & $90.53(17)$ & $90.80(6)$ & \\
\hline $\mathrm{Al}(1)-\mathrm{S}(1)-\mathrm{Al}(2)$ & & $138.16(6)$ & $150.52(3)$ & \\
\hline $\mathrm{C}(\mathrm{carb})-\mathrm{Al}(2)-\mathrm{S}(1)$ & & $92.93(12)$ & $\begin{array}{l}95.35(5), \\
110.55(5)\end{array}$ & \\
\hline $\mathrm{S}(1)-\mathrm{Al}(1)-\mathrm{S}(2)$ & & $96.71(6)$ & & \\
\hline $\mathrm{C}(\mathrm{carb})-\mathrm{Al}(1)-\mathrm{Al}(2)$ & $102.27(5)$ & & & \\
\hline $\mathrm{C}(\mathrm{carb})-\mathrm{Al}-\mathrm{H}(1)$ & & & & $99.5(7)$ \\
\hline
\end{tabular}




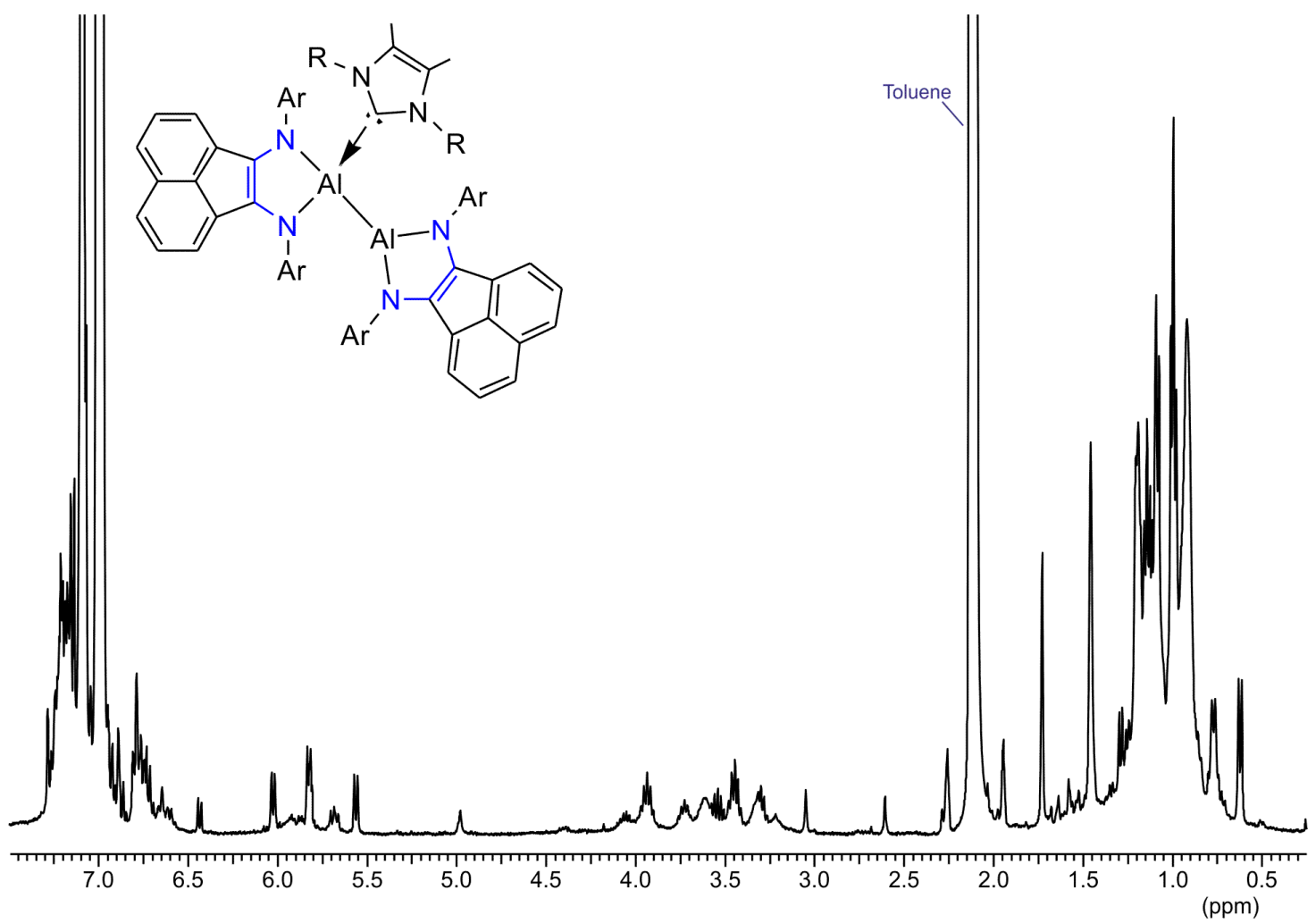

Fig. S1. ${ }^{1} \mathrm{H}$ NMR spectrum of complex 3 .

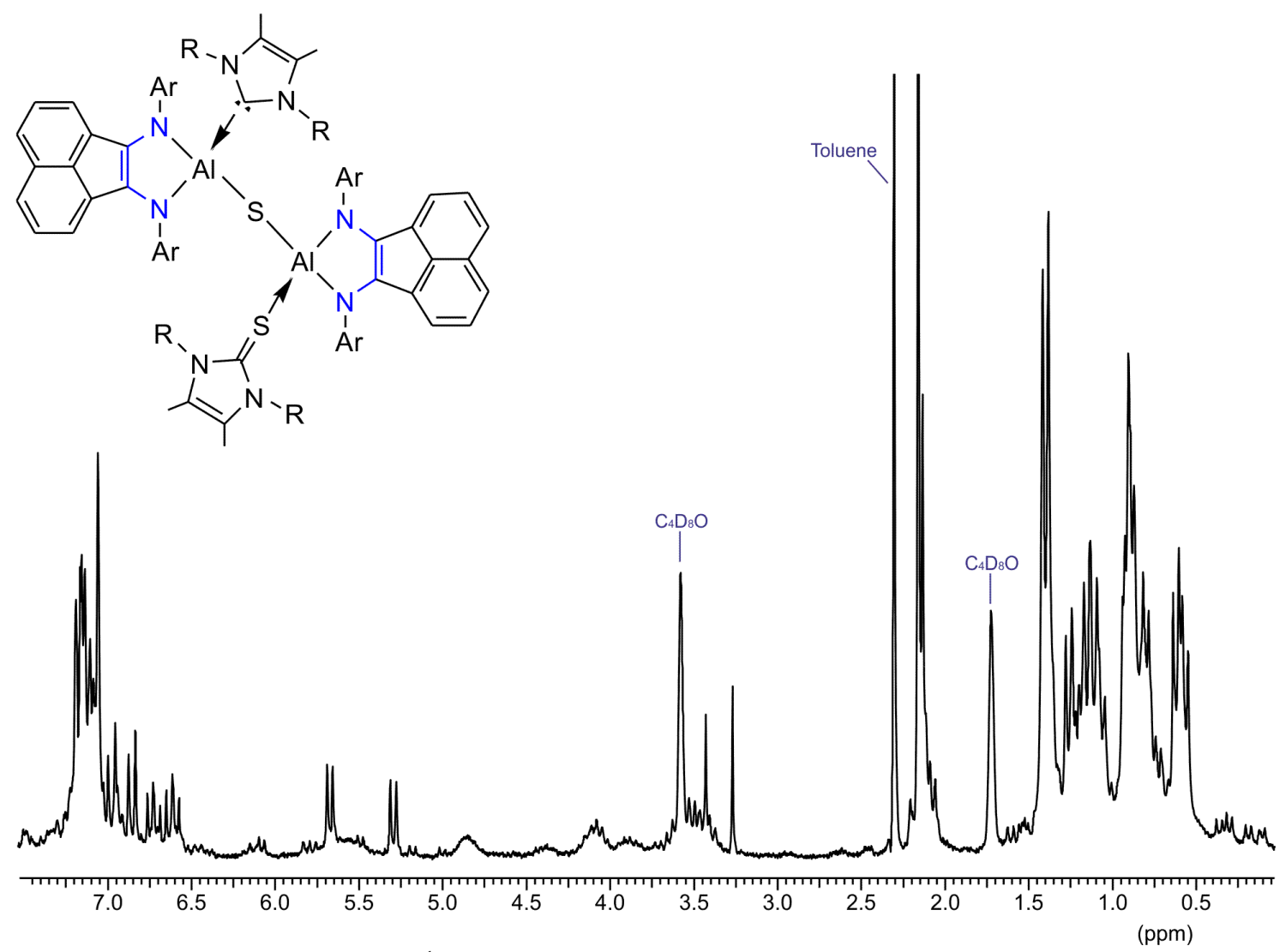

Fig. S2. ${ }^{1}$ H NMR spectrum of complex 4. 


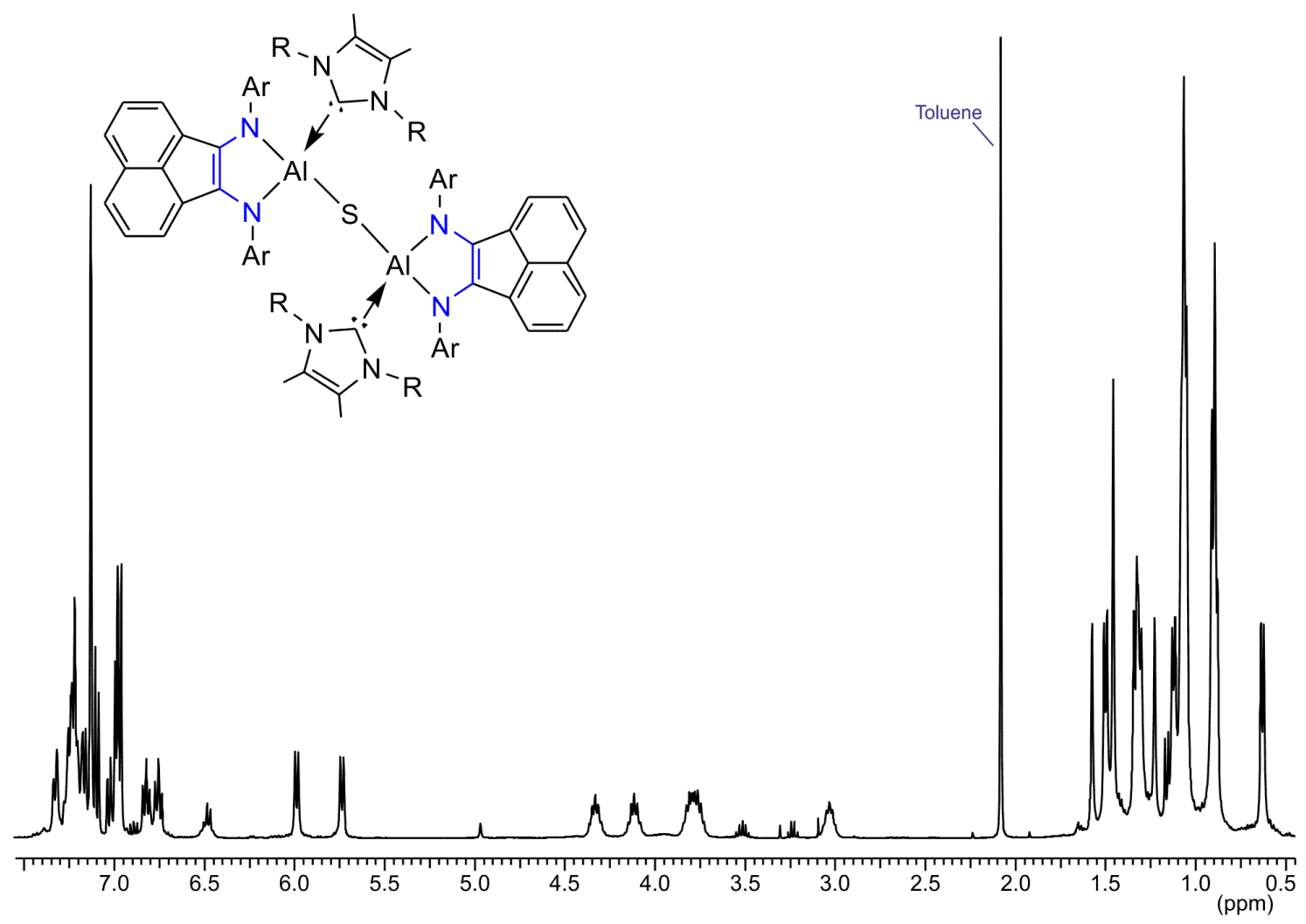

Fig. S3. ${ }^{1} \mathrm{H}$ NMR spectrum of complex 5.

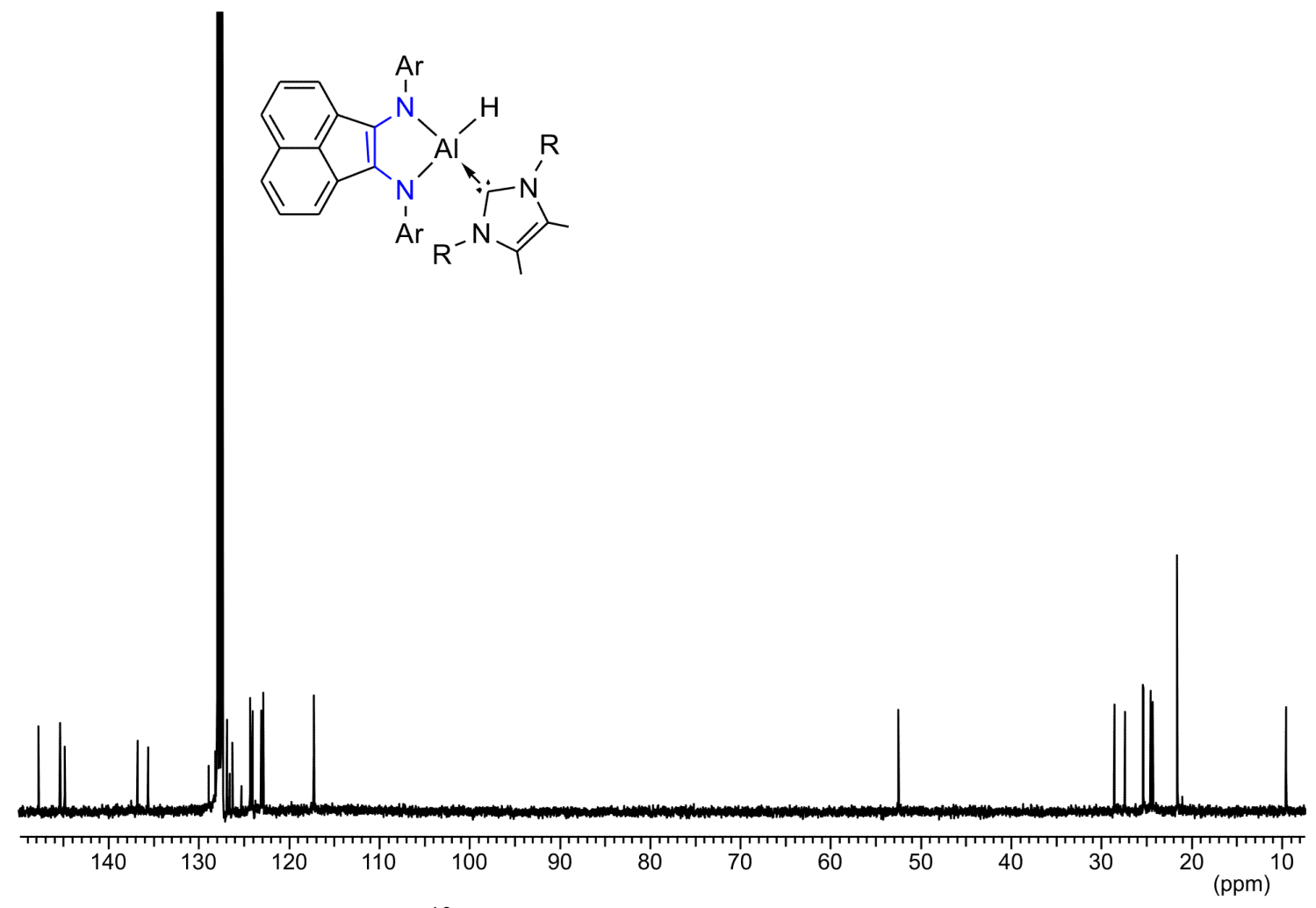

Fig. S4. ${ }^{13} \mathrm{C}$ NMR spectrum of complex 6 . 

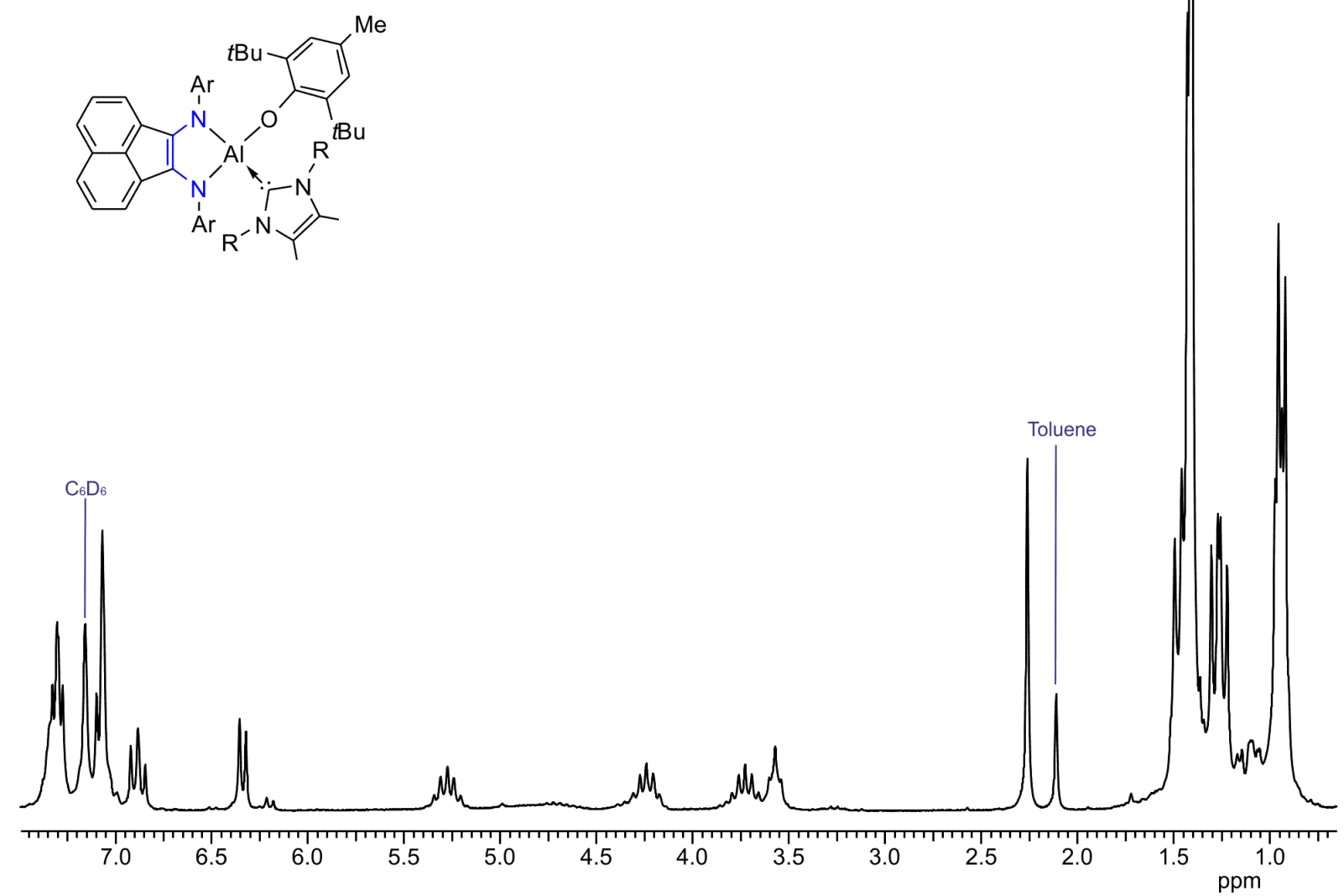

Fig. S5. ${ }^{1}$ H NMR spectrum of complex 7.

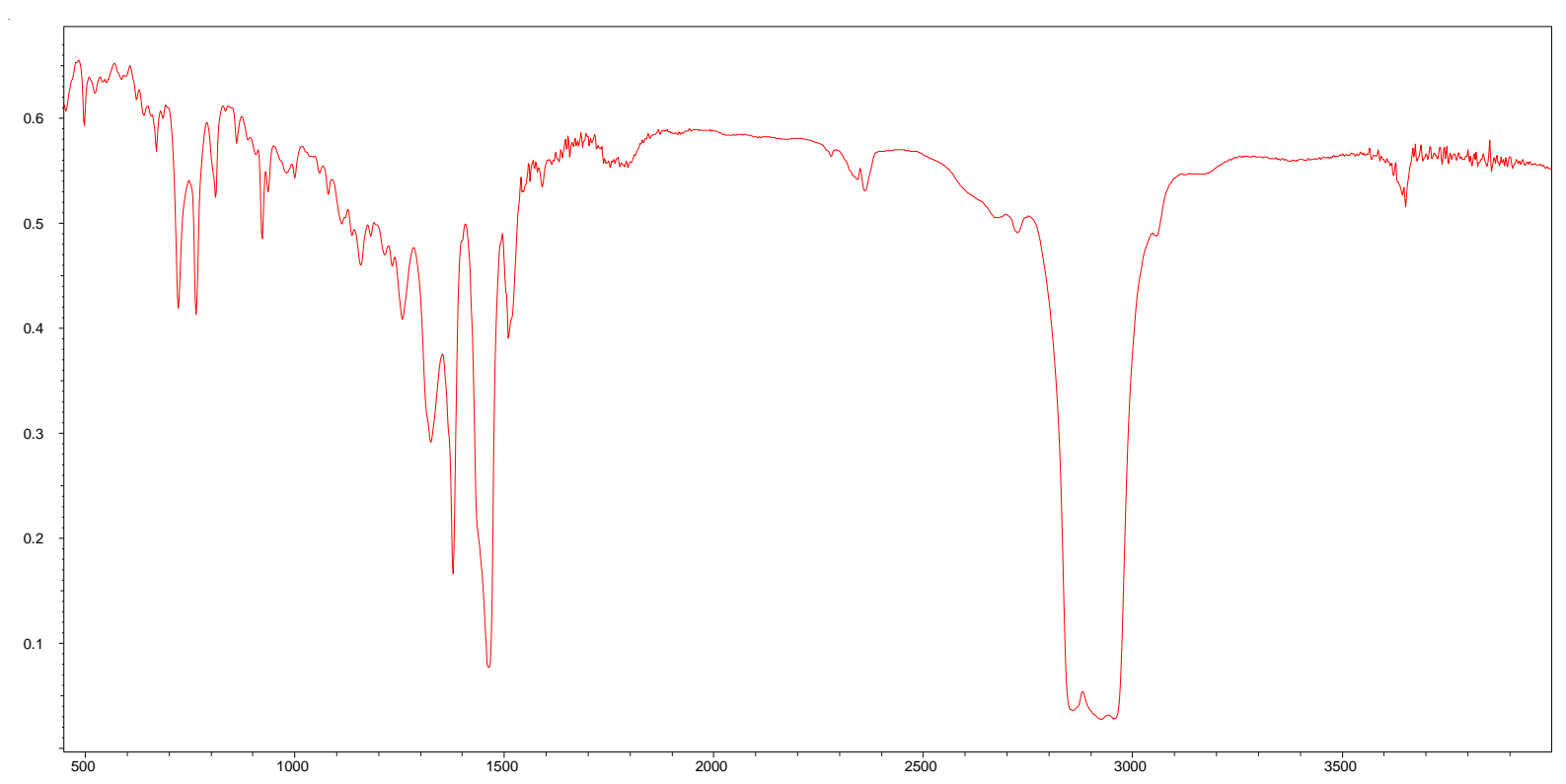

Fig. S6. IR spectrum of complex 7. 